\title{
A Chandra View of the Multi-scale Structures in Centaurus A
}

M. Karovska, G. Fabbiano, M. Elvis, F. Nicastro, S. S. Murray, and R. P. Kraft

Harvard-Smithsonian Center for Astrophysics, 60 Garden Street, Cambridge, MA 02138, USA

\begin{abstract}
Centaurus A (NGC 5128) is a giant early-type galaxy containing the nearest radio-bright AGN. The high-angular resolution Chandra images of Cen A reveal multi-scale X-ray structures with unprecedented detail and clarity. We present results from our study of X-ray structures surrounding the Cen A nuclear region, including the discovery of giant arc-like structures, extending to about $8 \mathrm{kpc}$ in the direction perpendicular to the jet. We compare the X-ray structures with observations in other wavelengths and discuss their implication for a global understanding of the morphology of Cen A.
\end{abstract}

\section{Introduction}

Centaurus A (Cen A) is a giant elliptical galaxy containing the nearest (at $3.5 \mathrm{Mpc}$; 1' $\sim 1 \mathrm{kpc}$ ) radio-bright Fanaroff-Riley Class I AGN. The nuclear region at the center of this giant elliptical galaxy is believed to be associated with a supermassive black hole, $M_{B H}=2 \times 10^{8} \mathrm{M}_{\odot}$, (Marconi et al. 2001). Because of its proximity, Cen A provides a unique laboratory for testing models of early galaxy evolution and structure and for studying the environment of quasar-like nuclei.

Multi-wavelength observations of Cen A, ranging from X-rays to radio, reveal its complex geometry (vis review by Israel 1998, and references therein). The most striking features in the optical and infrared images are several dark bands stretching across the middle of the galaxy, probably due to absorption by dust and other cool material. The dust lane as well as a set of faint concentric shell fragments and filaments extending to $16 \mathrm{kpc}$ from the nuclear region are probably remnants of a merger with a smaller spiral galaxy (Schiminovich et al. 1994). The radio continuum maps are dominated by a jet with huge radio lobes extending across the galaxy to several hundreds of kpc from the nucleus, while maps obtained in the $21 \mathrm{~cm}$ HI line show emission originating from cool material in two distinct regions of the galaxy.

It is very difficult to unify the different components of this galaxy and understand how they interact. Our recent discovery of kpc-scale X-ray arcs using Chandra could provide an important key for solving the puzzle. 


\section{X-ray Observations of Cen A}

X-ray observations of Cen A with Einstein and ROSAT lead to the detection of complex multi-scale emission structures in this galaxy. These include a bright nucleus, an extended X-ray jet and lobes, numerous point sources, and diffuse emission (Feigelson et al. 1981; Dobereiner et al. 1996)

Since Chandra's launch in 1999 Cen A was observed on several occasions using the HRC and ACIS instruments. The high angular resolution Chandra images reveal multiscale X-ray structures with unprecedented detail and clarity. Using Chandra observations detailed studies have been carried out of the Xray emission from the jet and the point source population (Kraft et al. 2001; 2002). Karovska et al. (2001) carried out sub-arcsecond resolution studies of the nuclear region using HRC-I images and deconvolution techniques and detected an extension similar in size and direction to the arc-second scale disk observed with HST/NICMOS in $\mathrm{Pa} \alpha$ line (Schreier et al. 1998).

The large-scale structures (several arcminutes in size) detected with Chandra suggest complex symmetry, including a component possibly associated with the inner radio lobes, and a separate component with an orthogonal symmetry that may be associated with the galaxy as a whole.

\section{Cen A X-ray Arcs}

We discovered a spectacular set of galaxy size arc-like structures in the diffuse emission in Cen A using archival Chandra (HRC-I and ACIS-I) observations. The arcs are symmetric about an axis perpendicular to the direction of the jet (Fig. 1). These faint arcs appear to be fragments of an ellipse and are consistent with a ring with an outer radius of $\sim 8 \mathrm{kpc}$ lying in a plane perpendicular to the radio jet (Karovska et al. 2002).

In Figure 1 we show a comparison of the spatial distribution of the X-ray emission (the central 16'x16' region from the smoothed HRC-I image) with the emission seen at radio wavelengths: the VLA $21 \mathrm{~cm}$ radio continuum map of the inner lobes of the jet (Condon et al. 1996), and $21 \mathrm{~cm}$ HI line emission map (van Gorkom 2002, priv. com.) (see also a color version http://chandra.harvard.edu/ photo/2002/0157/index.html). The NW X-ray arc appears to fill a region enclosed between the inner HI emission (central $4 \mathrm{kpc}$ region), and the NW optical and $\mathrm{HI}$ shells at a distance of about $8-10 \mathrm{kpc}$ from the center. Its outer boundary is surrounded by an arc of blue stellar emission. The somewhat fainter SE arc is similarly located between the inner HI emission and the outer optical shells in the SE. The thickness of these arcs is about $2 \mathrm{kpc}$. The arcs trace an ellipse with a semi-major axis of about $8 \mathrm{kpc}$, and a semi-minor axis is about 3-4 kpc. The geometry is consistent with a ring structure seen in a projection (inclination of 60-70 degrees from the plane of the sky) with an axis of symmetry along the direction of the inner jet.

Critical information on the nature of the X-ray arcs can be derived from the spectra. Unfortunately, the archival ACIS-I observations include only a fraction of the NW arc, and the SE arc is out of the field of view. Figure 2 shows the extracted spectrum in the region of the NW arc $(20 \mathrm{cnts} / \mathrm{bin})$. Our initial spectral analysis suggests that the X-ray emission could be thermal or a 


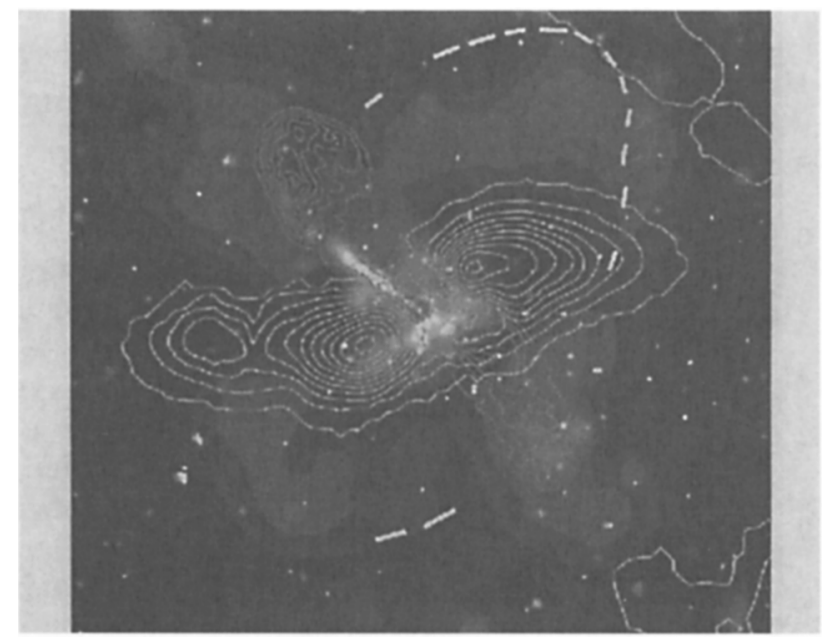

Figure 1. HRC-I X-ray image of the 16 ' $\times 16^{\prime}$ ' region centered on the nucleus with the contours of the radio continuum map of the inner jet lobes, and the radio HI gas emission. A dashed line is an outline of the optical arc. $\mathrm{N}$ is up and $\mathrm{E}$ is to the left.

result of a shock (Karovska et al. 2002). However, the best fit with a thermal model is strongly dependent on the initial assumptions, especially regarding the intrinsic equivalent hydrogen column density. The fit obtained by leaving the column density and the temperature free to vary, gives $k T=0.40 \mathrm{keV}$, and $N_{H}=3.9 \times 10^{21} \mathrm{~cm}^{-2}$, quite different from those obtained in the case with frozen $N_{H}$. Freezing the NH to $1 \times 10^{20} \mathrm{~cm}^{-2}$ (the lowest contour of the radio $\mathrm{H}$ I emission) increases the estimated temperature to $\sim 0.7 \mathrm{keV}$. This indicates that these parameters are strongly correlated to each other, and that the signalto-noise in the spectrum is insufficient to distinguish between any given model.

There are several possible explanations of the the nature and the origin of the arcs (Karovska et al. 2002; They may be apparent structures in the extended diffuse X-ray halo, a result of a varying density and local extinction, or the arcs may be bordering a region of interaction between the infalling gas from a tidal-tail and the material in the middle of the galaxy. However, the apparent symmetry of the ring and the alignment perpendicular to the radio jet seem to suggest an origin related to the nucleus.

More likely, the diffuse emission in the arcs (or the torus) is a result of an interaction between a powerful equatorial outflow (or wind) with the system of stellar shells or the ISM. A giant eruption, resulting for example from a transient nuclear activity, could have produced a galaxy-sized shock wave propagating in the plane perpendicular to the direction of the jet. The regions at the inner boundaries of the arcs could be evacuated cavities. By assuming that the X-ray energy in the torus is equal to the kinetic energy of the ejecta, we derive a mean velocity of $\sim 600 \mathrm{~km} / \mathrm{s}$. In the case of uniform expansion the outburst could have occurred few $10^{7}$ years ago. 

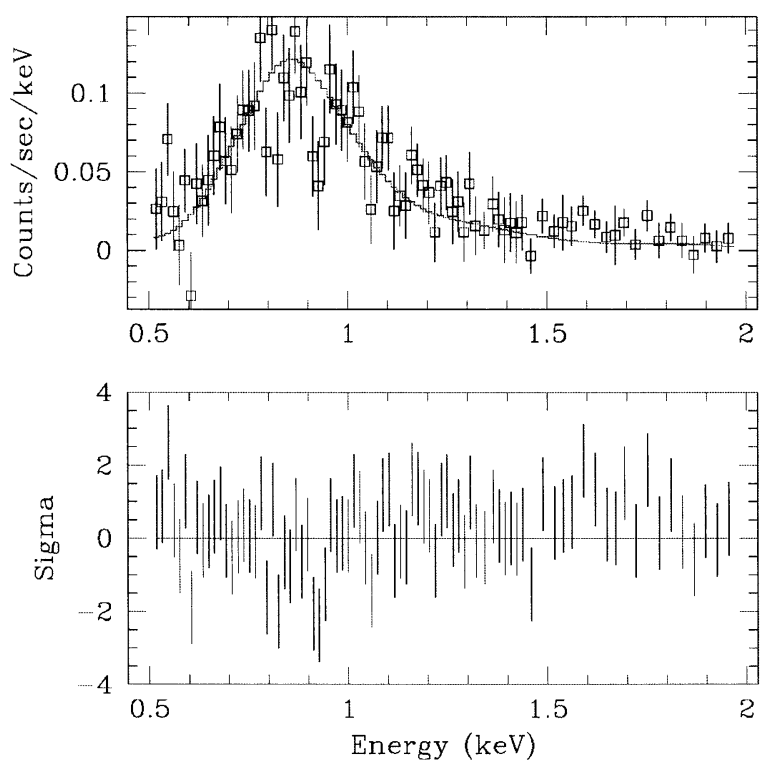

Figure 2. ACIS-I spectrum of the NW arc along with the best fitting thermal model (upper panel), and the residuals in $\sigma$ (lower panel).

To determine the true nature and the origin of the emission from the arcs in Cen A further multiwavelength observations are required, including obtaining high signal-to-noise spectra of the diffuse emission in both arcs as well as in the surrounding regions. This could result in a more detailed knowledge of the physical characteristics of these regions, including mapping of the temperature and the local absorption, and will provide a basis for discriminating between various hypotheses regarding the nature and the origin of the arcs.

Acknowledgments. This work was supported in part by NASA contract NAS8-39073 (Chandra X-ray Center) and a CXC travel grant.

\section{References}

Condon, J. J. et al. 1996, ApJS, 103, 81

Dobereiner, S. et al. 1996, ApJ, 470, L15

Feigelson, E. D. et al. 1981, ApJ, 251, 31

Israel, F. P. 1998, A\&AR, 8, 237

Karovska, M. et al. 2001, IAU Symp., 205, 45

Karovska, M. et al. 2002, ApJ, 577, 114

Kraft, R. P. et al. 2001, ApJ, 560, 675

Kraft, R. P. et al. 2002, ApJ, 569, 54

Marconi, A. et al. 2001, ApJ, 549, 915

Schreier, E. J. et al. 1998, ApJ, 499, L143

Schiminovich, D. et al. 1994, ApJ, 423, L101 